\title{
Relationship between Running Activity Rhythm and the Development of Activity-Stress Ulcer in Rats
}

\author{
Kouki WATANABE, Chiaki HARA and Nobuya OGAWA \\ Department of Pharmacology Ehime University School of Medicine. \\ Shigenobu-cho, Onsen-gun. Ehime 791-02, Japan \\ Accepted November 24, 1989
}

\begin{abstract}
In the present study, the relationship between the development of activity-stress (A-S) ulcer and the behavioral and physical changes of rats with the A-S paradigm was investigated. We applied the endoscopic technique to establish the A-S ulcer as a model for studying the therapeutic or healing process of ulcers. Male and female rats were subjected to daily $1 \mathrm{hr}$ feeding in the light (L)-phase (09:00-10:00) or in the dark (D)-phase (21:00-22:00). Prior to peak-revolution of running-activity, ulcer occurred earlier in the $L$-phase feeding group than in the D-phase feeding group. The ulcer development was accompanied with a change in the circadian rhythm of running-activity: e.g., the running-activity of the L.-phase was higher than that of the $D$-phase. This phenomenon was more marked in the L-phase feeding group than in the D-phase feeding group. In addition, the ulcer development was closely related to body weight loss. No sex differences were found in these phenomena. The results suggest that change in the circadian rhythm of running-activity, body weight loss and high running-activity are important variables for predicting the A-S ulcer production, avoiding the need to kill the animals to determine ulcer formation.
\end{abstract}

When young male rats are individually housed in activity-wheel cages and fed only 1 hr each day, they die within 4-12 days after beginning the restricted feeding schedule. These rats reveal extensive lesions in the glandular stomach (1). They also showed characteristic behavioral and physical changes such as excessive running-activity, severe body weight loss and low food consumption. Since only the rats that showed high runningactivity revealed gastric lesions, it was suggested that high running-activity was a essential etiological component of the ulceration (2). Paré and Houser called the gastric lesions "activity-stress (A-S) ulcer" (3).

On the other hand. Pare has proposed this ulcerogenic technique as a model for studying therapeutic procedures, because the A-S ulcer was alwavs observed even after 4 recovery days of ad lib feedings and no running (4). In order to utilize the A-S ulcer for this purpose. the development of gastric lesions must be determined without killing the animals before beginning the recovery period. We have used the endoscopic technique to determine the development of gastric lesions, but frequent endoscopic observations are difficult because the rats reveal not only gastric lesions but also exhaustion as indicated by metabolic imbalance (5) and immunodeficiency (6). According!y, behavioral markers for predicting the development of gastric lesions are needed. Paré has advocated the following three signs as markers (4): 1) a large initial drop in the body weight. 2) a rapid decline in daily food consumption and 3) a drop in wheel-activity from the peak level. Because the rats were already in a state of exhaustion when the gastric lesions were confirmed using these three markers, it is necessary to determine the develooment of gastric lesions in an earlier phase. Hara et al. (7) have reported that when the rats were subjected to a daytime feeding schedule, only the animals with a reversal of circadian rhythm of running-activity synchronizing with feeding time revealed 
gastric lesions. Therefore, we assumed that a change in the circadian rhythm of runningactivity might be useful as an additiona! marker to predict the development of gastric lesions in an earlier phase

From these points of view, the present study investigated the relationship between the development of gastric lesions and circadian rhythm of running-activity and behavioral and physical changes of male and female rats.

\section{Materials and Methods}

Animals: Forty male and forty female Wistar strain rats, at 9 weeks of age were used. Prior to the experiment, the rats were housed in polycarbonate cages, four animals per cage. The room temperature was controlled at $23 \pm$ $1{ }^{\circ} \mathrm{C}$ under a 12:12 LD cycle (lights on 07:0019:00).

Apparatus: The activity-wheel cage consists of a standard running-wheel made of stainless steel $(33 \mathrm{~cm}$ in diameter, $11 \mathrm{~cm}$ wide). Each activity wheel also included an adjoining cage measuring $40 \times 15 \times 15 \mathrm{~cm}$, in which the rats took food and water ad libitum. A sliding door could separate the wheel from the adjoining cage; and when this door was opened, the rats could enter the running wheel freely. Control rats remained only in the adjoining cage throughout the experiment. All wheel-revolutions were individually recorded for each activity cage using a photosensor in an one hour interval by a micro-computer system (NEC model PC9801 VX21: Biomedica, Ltd., Model BCS-1305).

Procedure: The animals were randomly divided into two groups, the activity-wheel cage housing group (activity group) and the adjoining cage housing group (control group). Then each group was randomly subdivided into two groups: the light phase $1 \mathrm{hr}$ feeding group (L-group: feeding time 09:00-10:00) and the dark phase 1 hr feeding group (D-group: feeding time 21:00-22:00). Each group consisted of 10 animals. All animals were individually housed and allowed to adapt to the housing conditions for 3 days under free access to food and water. During the 3-day adaptation period, the sliding door was closed to prevent the rats from entering the running wheel. The next day
(Day 1), food was withdrawn at 10:00 in the L-group and at 22:00 in the D-group, and the 1 hr feeding schedule was started. The activity group was allowed to enter freely into the running-wheel from the same day (Day 1) by keeping the sliding door open. Daily running-activity and the \% ratio of light phase activity/whole day activity (L/(L.+D)\%) were automatically recorded daily. Measurement of body weight and food consumption and endoscopic observation were performed daily between 00:00-02:00 in the L-group or between 12:00-14:00 in the D-group throughout the experiment. The endoscopic observation was performed with an angle-manipulatable micro-fiber scope with a diameter of $2.7 \mathrm{~mm}$ (Olympus K.K., Model PF-27 $\mathrm{M}$ and Model CLE-F). The procedure for endoscopic observation was in accordance with that of the previous report (8). If a rat died, the stomach was removed and inspected for lesions to record the number and cumulative length of lesions. The experiment was continued for 14 days; at this time all surviving rats were sacrificed with ethyl-ether, and their stomachs were removed and inspected for lesions.

Statistics: Since a large number of animals in the activity group died during the experiment, data from daily changes of body weight and food consumption were assessed on each day by Kruskal-Wallis one-way analysis of variance followed by the nonparametric Tukey test and data from daily changes of wheel-revolutions and $L /(L+D) \%$ were assessed on each day by Mann-Whitney's $U$ test. The surviva! times were analyzed by Kaplan-Meier's product-limit test. The difference of the survival time between the control and the activity groups or between the $L$ - and D-groups in both sexes were evaluated with the generalized Wilcoxon test. The number of days for development of gastric lesions were evaluated by Kruskal-Wallis oneway analysis of variance followed by the nonparametric Tukey test. The stomach pathology was evaluated by Mann-Whitney's $U$-test. In the comparison between two related samples in the Day 1 group, the day before G-day group (G-day means the day when gastric lesions were found with a micro-fiber endoscope) and the G-day group 
were evaluated with the Wilcoxon matchedpairs signed-ranks test.

\section{Results}

Only in the activity group. all of the rats revealed gastric lesions, and most of them died by the end of this experiment. Among the male rats, the survival times of the L-group and the D-group were 9.5 days and 10.8 days, respectively: and among the female rats, the survival times were 10.0 days or 9.8 days, respectively. There were significant differences between the control and the activity groups in the survival time ( $L$-group: in males, $Z=4.0, P<0.01$; in females, $Z=4.0, P<0.01$; $D$-group: in males, $Z=4.0, P<0.01$; inf emales, $Z=3.9 . \quad P<0.01$ ). However, no sex and feeding time differences were found in the survival time.

Figure 1 illustrates the mean daily body weight during the experiment. After food restriction was started, the body weights in all groups decreased throughout the experiment. There were significant differences among the four experimental groups each day among both males and females: males: Day $1(\mathrm{H}=$ 8.60, $\mathrm{P}<0.05)$. Day $2(\mathrm{H}=15.46, \mathrm{P}<0.01)$, Day $3(H=18.13, P<0.01)$, Day $4(H=18.76$, $P<0.01)$, Day $5(H=25.06, P<0.01)$, Day 6
$(\mathrm{H}=24.10, \mathrm{P}<0.01)$, Day $7 \quad(\mathrm{H}=26.44, \mathrm{P}<$ $0.01)$. Day $8(H=24.29, P<0.01)$, Day 9 $(\mathrm{H}=25.18, \mathrm{P}<0.01)$. Day $10(\mathrm{H}=22.86, \mathrm{P}<$ $0.01)$. Day $11(H=18.11, P<0.01)$. Day 12 $(H=13.53, P<0.01)$, Day $13(H=11.58 . P<$ $0.01)$ : females: Day $3(H=11.66, P<0.01)$, Day $4(H=10.58, P<0.05)$, Day $5(H=16.11$. $P<0.01)$. Day $6(H=17.41, P<0.01)$. Day 7 $(H=23.54, P<0.01)$, Day $8(H=21.51, P<$ $0.01)$. Day $9(H=22.07, P<0.01)$. Day 10 $(H=21.98, P<0.01)$, Day $11 \quad(H=19.85, P<$ $0.01)$. Day $12(H=19.77, P<0.01)$. Day 13 $(H=16.41, P<0.01)$. Day $14(H=13.78, P<$ 0.01 ). In both males and females, body weight loss was significantly greater for the activity group than the control group. However, there was no significant difference between the $L$ and the D-groups.

Figure 2 illustrates daily food consumption in each group. The food consumption on Day 1 markedly decreased as compared with the pre-level. In the activity group, daily food consumption increased daily until the peak level. Following the peak level, the food consumption decreased day by day. In the control group, food consumption was stable ranging between $8-10 \mathrm{~g}$ in males and between $6-8 \mathrm{~g}$ in females. There were significant differences among the four experimental groups each day
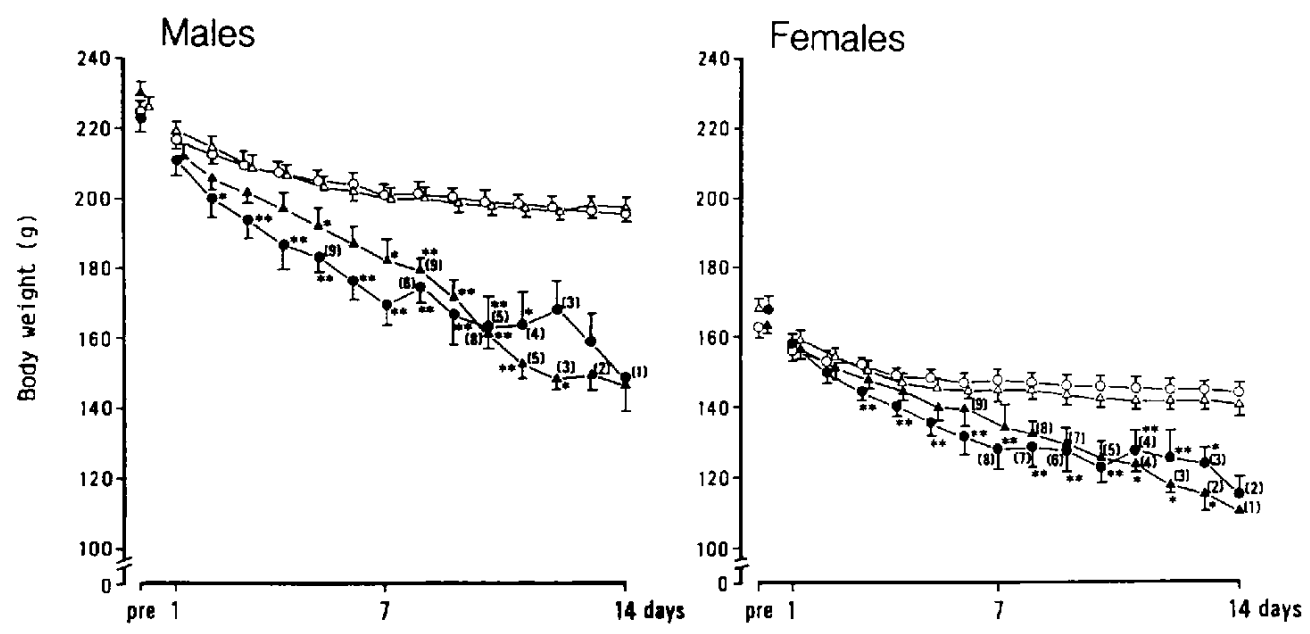

Fig. 1. Mean ( \pm S.E.M.) daily body weight changes of rats exposed to the 1 hr restricted feeding conditions. Numbers in parentheses indicate the number of rats represented by a particular data point. " $P<0.05, * * P<0.01$ : Statistical difference between the control and the activity groups (nonparametric Tukey test). (O): L-phase fed/control group, $(\triangle)$ : D-phase fed/control group, (O): L-phase fed/ activity group, (A): D-phase fed/activity group. 

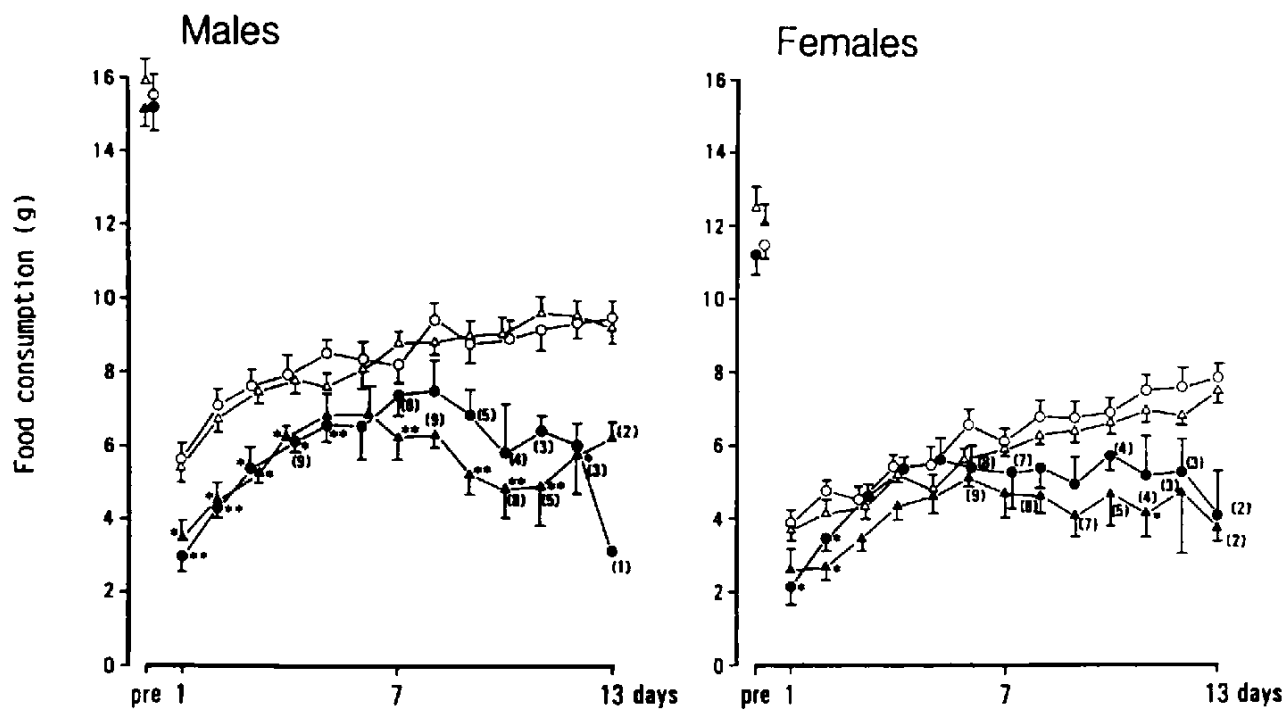

Fig. 2. Mean ( \pm S.E.M.) daily food consumption of rats exposed to the $1 \mathrm{hr}$ restricted feeding conditions. Numbers in parentheses indicate the number of rats represented by a particular data point. ${ }^{*} P<0.05$. "P $P<0.01$ Statistical difference between the control and the activity group (nonparametric Tukey test). (O): L-phase fed/control group. ( $\triangle$ ): D-phase fed/control group. (O): L-phase fed/activity group, (A): D-phase fed/activity group.

in both the males and females: in males: Day 1 $(H=18.97, P<0.01)$, Day $2(H=21.47, P<$ $0.01)$. Day $3(H=17.96, P<0.01)$. Day $4(H=$ 15.98, $P<0.01)$, Day $5(H=15.61, P<0.01)$. Day $7(H=12.06, P<0.01)$. Day $8(H=14.17$. $P<0.01)$. Day $9(H=19.92, P<0.01)$, Day 10 $(H=19.43, P<0.01)$, Day $11(H=13.60, P<$ 0.01). Day $12(H=15.39, \quad P<0.01)$; in females: Day $1(H=13.19, P<0.01)$, Day 2 $(H=21.88, P<0.01)$, Day $3 \quad(H=9.76, P<$ $0.05)$. Day $4(H=9.01, P<0.05)$, Day $8(H=$ 11.13, $P<0.05)$, Day $10(H=9.28, P<0.05)$. Day $11(\mathrm{H}=12.58, \mathrm{P}<0.01)$, Day $12(\mathrm{H}=$ 7.93, $P<0.05)$. Day $13(H=11.02, P<0.05)$. Daily food consumption of the activity group was significantly lower than that of the control group. However, there was no difference between the L-and the D-groups in daily food consumption.

Figure 3 illustrates the mean daily runningactivity. After food restriction was started. daily wheel-revolutions of the activity group was gradually increased, and after achieving peak revolution, it began to decrease rapidly. The number of wheel-revolutions in the early phase was significantly greater for the $\mathrm{L}$ group than the D-group. In this phase, the running-activity of the females tended to be higher than that of males.

Figure 4 shows daily changes in the circadian pattern of running-activity in the Lgroup and the D-group. Data were represented with \% ratio of light phase activity / whole day activity $(\mathrm{L} /(\mathrm{L}+\mathrm{D}) \%) . \mathrm{L} / \mathrm{L}(\mathrm{L}+\mathrm{D}) \%$ of the L-group was significantly higher than that of the D-group, both in males and females. $L /(L+D) \%$ of the $L$-group in males or females was over $50 \%$ from Day 5 or Day 6 . On the contrary, $L /(L+D) \%$ of the $D$-group in both males or females was over $50 \%$ from Day 11 or Day 13. This means that the runningactivity during the L-phase in the L-group gradually increased, and the nocturnal rhythm of the running-activity of the L-group was disrupted earlier than that of the D-group.

Figure 5 shows the endoscopic appearances of the gastric mucosa of representative rats in the four activity groups on Day 1, the day before G-day, and the day after G-day. As shown in Fig. 5, gastric lesions that were presented on $\mathrm{G}$-day became more severe on the day after. On the contrary, all control groups showed no gastric lesions throughout the experiment. On the basis of the daily en- 

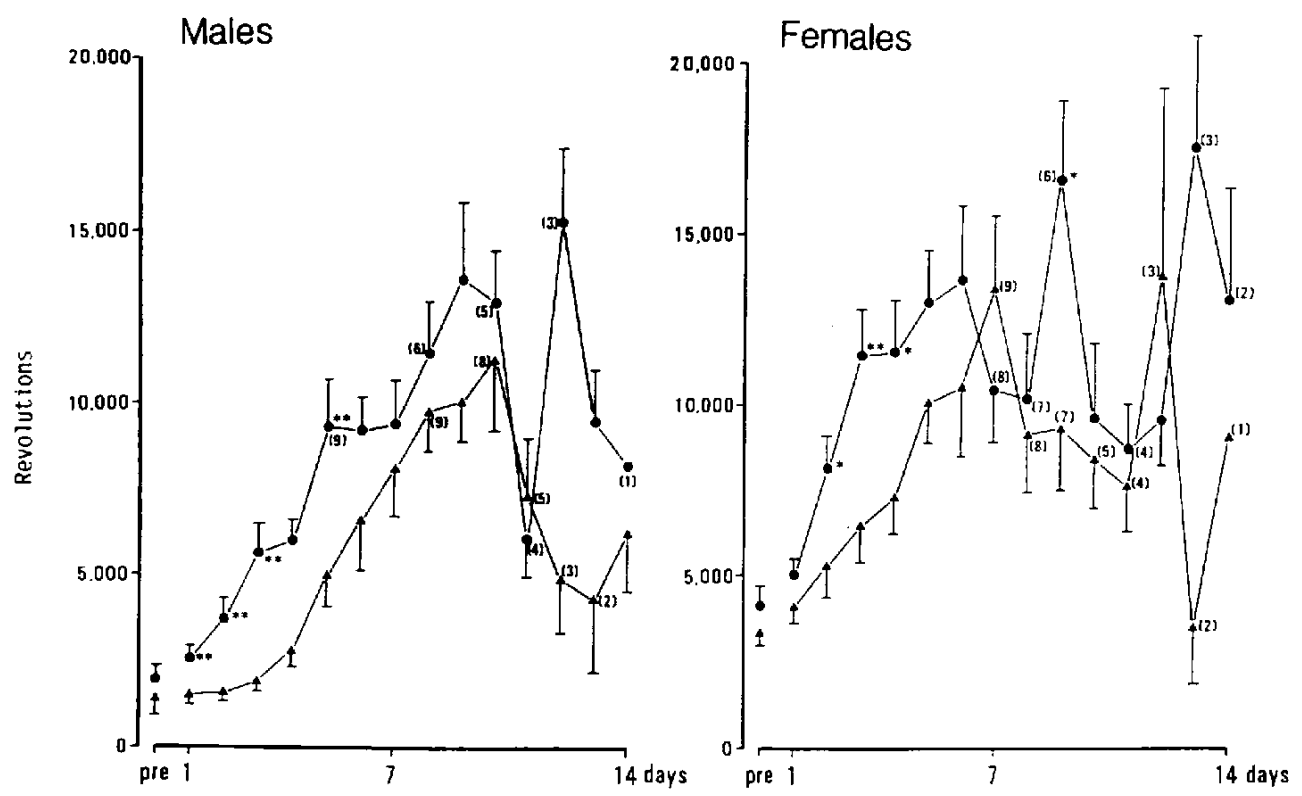

Fig. 3. Mean ( \pm S.E.M.) daily running-activity of rats exposed to the $1 \mathrm{hr}$ restricted feeding conditions. Numbers in parentheses indicates the number of rats represented by a particular data point. ${ }^{*} P<0.05$, *P<0.01: Statistical difference between the L- and the D-groups (Mann-Whitney's $U$-test). L-phase fed/activity group, (A): D-phase fed/activity group.
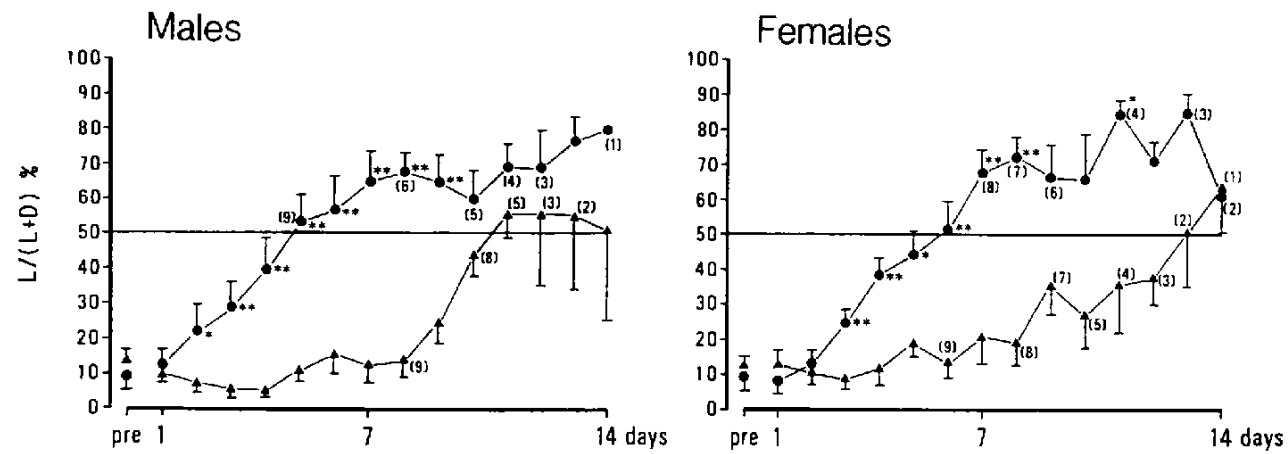

Fig. 4. Mean ( \pm S.E.M.) daily $L /(L+D) \%$ of rats exposed to the 1 hr restricted feeding conditions. Numbers in parentheses indicate the number of rats represented by a particular data point. ${ }^{*} \mathrm{P}<0.05$. ${ }^{*} \mathrm{P}<0.01$ : Statistical difference between the $\mathrm{L}$ - and the D-groups (Mann-Whitney's $U$-test).

L-phase fed/activity group, (A): D-phase fed/activity group.

doscopic observation, the number of days for development of gastric lesions was investigated. The results are shown as follows: L-group: males $=6.7 \pm 1.0$ days (mean \pm . S.E.M.), females $=6.5 \pm 0.9$ days; $D$-group: males $=8.8 \pm 0.5$ days, females $=7.9 \pm 0.5$ days. There was a significant difference among the four experimental groups $(\mathrm{H}=8.16, \mathrm{P}<0.05)$.
The development of gastric lesions of the Lgroup was significantly earlier than that of the D-group $(P<0.05)$ in males.

Table 1 indicates the stomach pathology in each experimental group. Only the activity group had lesions, and these were presented in the glandular portion of the stomach. There were no significant sex and feeding 


\section{Light phase feeding group}

Males

Rat $\# 3$

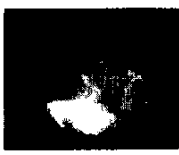

Rat \#5

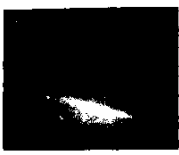

Fema les

Rat $\# 2$
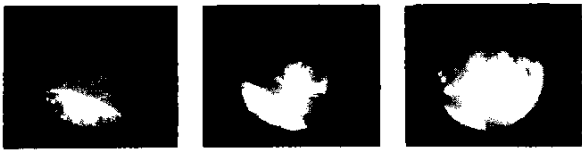

Rat \#9
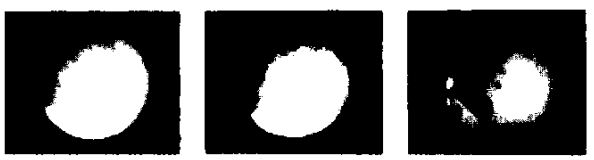

\section{Dark phase feeding group}

Males

day 1

the day
before G-day

G-day
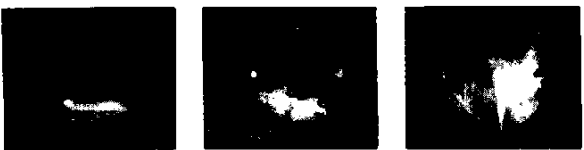

Rat \#1
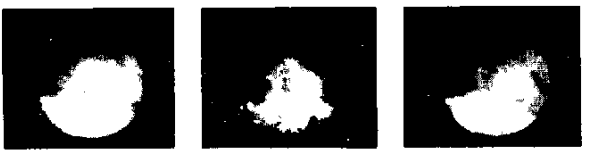

Rat $\# 5$

Females

Rat $\# 2$
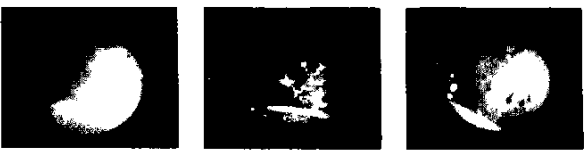

Rat $\# 8$
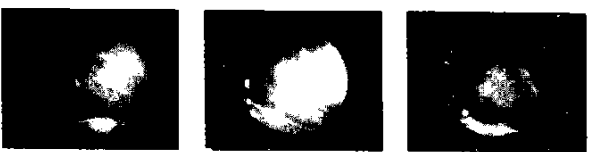
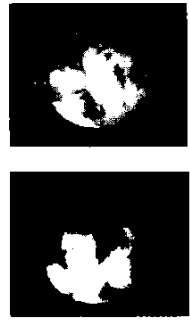

the day after G-day
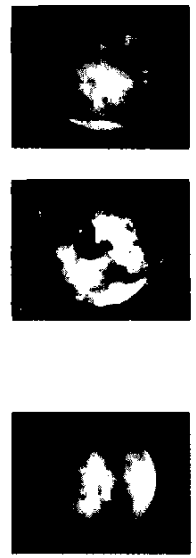

ter G-day
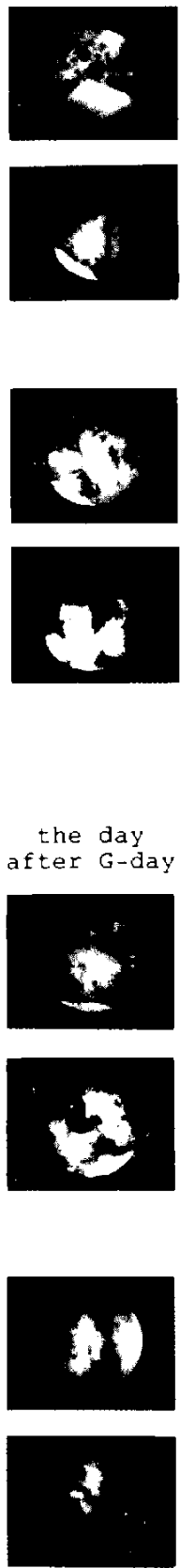

Fig. 5. Endoscopic appearances of the gastric mucosa of representative rats on Day 1 , the day before G-day ( $G$-day means the day when gastric lesions were found by using a micro-fiber endoscope). $G$-day and the day after $\mathrm{G}$-day. 
Table 1. Summary (MeantS.E.M.) of stomach pathology for all treatment groups

\begin{tabular}{|c|c|c|c|c|c|c|}
\hline & \multicolumn{3}{|c|}{ Activity group } & \multicolumn{3}{|c|}{ Control group } \\
\hline & $\begin{array}{l}\text { Rats with } \\
\text { lesions }\end{array}$ & $\begin{array}{l}\text { Nuimber of } \\
\text { lesions }\end{array}$ & $\begin{array}{c}\text { Cumulative } \\
\text { lesion length } \\
(\mathrm{mm})\end{array}$ & $\begin{array}{l}\text { Rats with } \\
\text { lesions }\end{array}$ & $\begin{array}{l}\text { Number of } \\
\text { lesions }\end{array}$ & $\begin{array}{l}\text { Cumulative } \\
\text { lesion length } \\
(\mathrm{mm})\end{array}$ \\
\hline \multicolumn{7}{|l|}{ Males } \\
\hline L-group $(n=10)$ & 10 & $15.6 \pm 3.0$ & $25.3 \pm 6.1$ & 0 & - & - \\
\hline D-group $(n=10)$ & 10 & $13.7 \pm 2.4$ & $20.7 \pm 4.6$ & 0 & - & - \\
\hline \multicolumn{7}{|l|}{ Females } \\
\hline L-group $(n=10)$ & 10 & $19.0 \pm 2.6$ & $34.3 \pm 5.4$ & 0 & - & - \\
\hline D-group $(n=10)$ & 10 & $15.9 \pm 3.1$ & $21.8 \pm 3.7$ & 0 & - & - \\
\hline
\end{tabular}

Statistical evaluations were performed by Mann-Whitney's $U$-test between the L-group and D-group and between male and female rats.

\section{Light phase feeding group}

Males
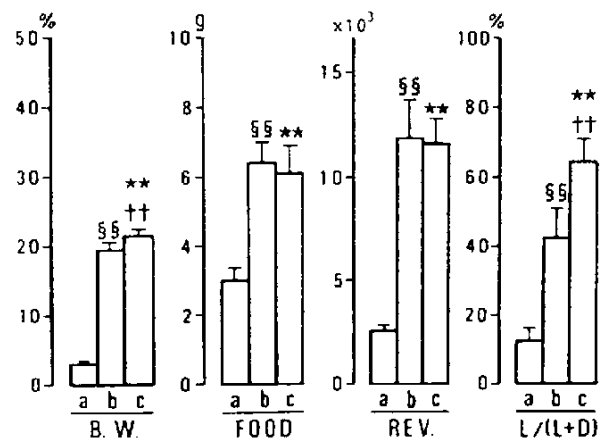

Females

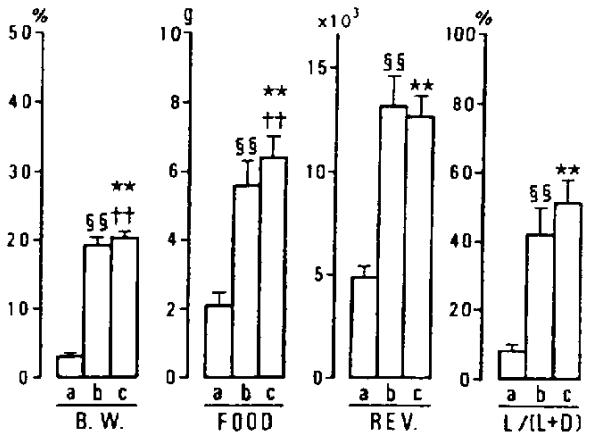

\section{Dark phase feeding group}

Males
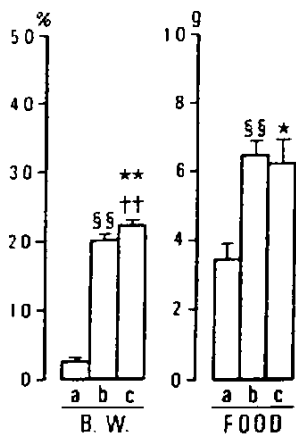
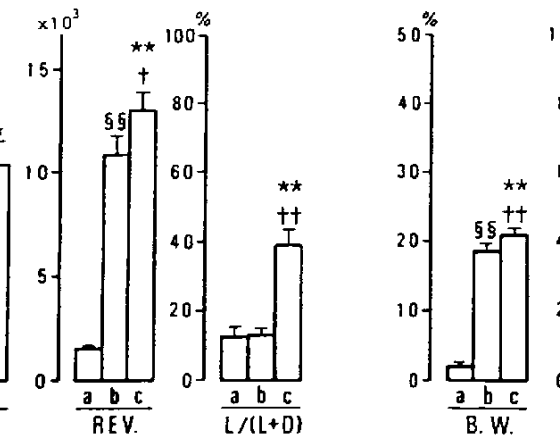

Females

Fig. 6. Changes (Mean \pm S.E.M.) of $\%$ body weight loss, daily food consumption, daily runring-activity and $L /(L+D) \%$ on Day 1 , the day before $G$-day ( $G$-day means the day when gastric lesions were found by using a micro-fiber endoscopel and $\mathrm{G}$-day. $\star P<0.05, \star \star P<0.01$ : Statistical difference between Day 1 and $G$-day; $T P<0.05$, t $P<0.01$ : between the day before $G$-day and $G$-day: $\$ \$ P<0.01$ : between Day 1 and the day before G-day (Wilcoxon matched-pairs signed-ranks test). a: Day 1; b: the day before G-day; $c:$ G-day. B.W.: \% body weight loss; FOOD: food consumptions per day: REV.: wheel-revolutions per day: $L /(L+D): \%$ ratio of light phase activity/whole day activity. 
time differences in either the number or cumulative length of lesions.

Figure 6 shows comparisons between $G$ day and Day 1 or the day before G-day in \% body weight loss, food consumption, runningactivity, and $L /(L+D) \%$ in the four activity groups. Body weight on G-day significantly decreased as compared with that on Day 1 or the before G-day. There were no sex and feeding time differences in $\%$ body weight loss. Food consumption on G-day significantly increased from Day 1 , but not from the day before $G$-day except for females of the $L$ group. Wheel-revolutions on G-day significantly increased as compared with that on Day 1. There was a difference between the L-group and the D-group in wheel-revolutions. That is, whee!-revolutions of the Dgroup on G-day increased as compared with the day before $G$-day, whereas that of the $L$ group on G-day were not different from the day before $\mathrm{G}$-day. There was no sex difference in $L /(L+D) \%, L /(L+D) \%$ on $G$-day was significantly higher than that on Day 1 or the day before $G$-day, except in females of the $L$ group. $L /(L+D) \%$ on the day before $G$-day was significantly higher than that on Day 1 in the L-group, but not in the D-group. That is, $L /(L+D) \%$ of the D-group was significantly increased just on G-day as compared to those of the previous days.

\section{Discussion}

The goal of the present study was to establish A-S ulcer as a model for studying therapeutic or healing processes of gastric ulcers. The possibility for establishing this model was supported by Paré's report in which an A-S ulcer was always observed even after four recovery days of ad lib feeding and no running (4). However, when the three behavioral and physical signs that were proposed by Paré (see the Introduction) appeared, the animals were already in a state of exhaustion. In this study, we searched for a new additional marker for predicting $A-S$ ulcer production before the animals entered a state of exhaustion. So, we investigated the time-dependent change of body weight. food consumption, wheel-revolutions, $L /(L+D) \%$ and gastric mucous under the A-S procedure.

Daily changes of body weight, food con- sumption and running-activity of the activity group in the present study were in accordance with those in previous reports $(2,4)$. There were no sex differences in the general patterns of these three variables except for the running-activity in the early phase. Only the running-activity level among the three variables showed a significant difference when the values of the $L$ - and $D$-groups were compared (Fig. 3). The L-group males developed gastric lesions earlier than the Dgroup. The $L /(L+D) \%$ of the $L$-group was high, whereas that of the D-group was still low (Fig. 4). This result is considered to be depend upon synchronization of activity rhythm with feeding time as described previously $(9-11)$. These results suggest that the $1 \mathrm{hr}$ feeding in the L-phase is a more stressful condition as compared with that in the Dphase.

The most prominent finding in this study is that there is a relationship between the change of circadian activity rhythm and the development of A-S lesions. In Fig. 6, behavioral and physical characteristics on Day 1 , the day before G-day and G-day are explained, and the importance of the running-activity level of the $\mathrm{L}$-phase is pointed out. In males, $\mathrm{L} / \mathrm{C}+$ D) $\%$ on G-day was significantly higher than that on the day before G-day in both the Lgroup and the D-group. In females, this phenomena was found only in the D-group. Thus, the development of the gastric lesions was in accordance with an increase of the running-activity of the L-phase, except for females in the $L$-group. These results suggest that $\mathrm{L} /(\mathrm{L}+D) \%$ is closely related to the development of stomach lesions. In addition, we may easily judge the critical point of ulceration, at which $L /(L+D) \%$ is over $50 \%$. These findings suggest that males of the $L$ group have an advantage over the other groups when investigating the development of A-S ulcer.

Regarding the predictability of the development of A-S lesions, Paré has advocated that three behavioral and physical markers such as body weight loss, food consumption and wheel-revolutions are essential (4). The results of the present study suggest that body weight loss seems to be most closely related with the development of 
gastric lesions. On the contrary, there was no marked change in food consumption for developing the A-S lesions. High runningactivity over 13.000 revolutions per day seems to be essential for the development of A-S ulcer. In addition, we consider that the increase of $L /(L+D) \%$ also seems to be closely related with the development of gastric lesions. This proposal is supported by the following facts: (a) rats exhibited the A-S lesions accompanied by a change of circadian running activity rhythm before they entered a state of exhaustion, (b) $L /(L+D) \%$ of the $L$. group was significantly higher than that of the D-group, (c) gastric lesions of the L-group developed sooner than that of the D-group. (d) $L /(L+D) \%$ on G-day significantly increased from the day before G-day, excent for in females of the L-group. From these facts, we are sure that change of circadian activity rhythm is an additional marker for predicting the development of A-S lesions.

In conclusion, in order to predict the development of A-S lesions, it is important to use male rats with a $1 \mathrm{hr}$ feeding schedule in the L-phase. Additionally, it is necessary to use 1) body weight loss, 2) high runningactivity and 3 ) change of circadian rhythm of running-activity as behavioral markers for predicting the developmet of A-S lesions. There were no sex differences in the results of the present study except for the running-activity. Thus, the present study suggests that the A-S ulcer model can be used as an ulcer model for studying healing or therapeutic processes of gastric ulcer by means of the endoscopic technique and the behavioral and physical markers described above.

Acknowledgments: We are grateful to Dr. Shigehiro Ohdo and Dr. Shigeyuki Nakano of the Department of Pharmacology, Ehime University School of Medicine for their critical reading and stimulating discussions on an earlier version of this manuscript.

\section{References}

1 Barboriak, J.J. and Krioblock, H.W., Jr.: Gastric lesions in food-restricted young rats. Proc. Soc. Exp. Biol. Med. 141, 830-832 (1972)

2 Paré, W.P.: The influence of fond consumption and running activity on the activity-stress ulcer in the rat. Am. J. Dig. Dis. 20, 262-273 (1975)

3 Paré, W.P. and Houser, V.P.: Activity and foodrestriction effects on gastric glandular !esions in the rat: The activity-stress ulcer. Bull. Psychon. Soc. 2, 213-214 (1973)

4 Paré, W.P.: Activity-stress ulcer in the rat: Frequency and chronicity. Physiol. Behav. 16, 699-704 (1976)

5 Paré, W.P.: Body temperature and the activitystress ulcer in the rat. Physiol. Behav. 18, 219$223(1977\rangle$

6 Hara, C., Ogawa, N. and Imada, Y.: The activitystress ulcer and antibody production in rats. Physiol. Behav. 27, 609-613 (1981)

7 Hara, C., Manabe, K. and Ogawa, N.: Influence of activity-stress on thymus, spleen and adrenal weights of rats: Possibility for an immunodeficiency model. Physiol. Behav. 27, 243-248 (1981)

8 Pack, S.J.: Gastric mucosal lesions in rats induced by the communication box paradigm: Experimental conditions, endoscopic abservation and effect of vagotomy. Folia Pharmacol. Japon. 83, 467-478 (1984) (Abs. in English)

9 Edmonds, S.C. and Adler, N.T.: Food and light as entrainers of circadian running activity in the rat. Physiol. Behav. 18, 915-919 (1977)

10 Rosenwasser, A.M., Pelchat, R.J. and Adler, N.T.: Memory for feeding time: Possible dependence on coupled circadian oscillators. Physiol. Behav. 32, 25-30 (1984)

11 Richter, C.P.: Sleep and activity: their relation to the 24-hour clock. In Sleep and Altered States of Consciousness. Edited by Ketty. S.S.. Evarts, E.V. and Williams, H.L., p. 8-29, Williamis and Wilkins Press, Baltimore (1967) 